\title{
Site-selective palladium-catalyzed oxidation of glucose in
}

\section{glycopeptides}

Niels R.M. Reintjens, ${ }^{1, \#}$ Liubov Yakovlieva, ${ }^{1, \#}$ Nittert Marinus, ${ }^{1}$ Johan Hekelaar, ${ }^{1}$ Francesca

Nuti, ${ }^{2}$ Anna Maria Papini, ${ }^{2}$ Martin D. Witte, ${ }^{1}$ Adriaan J. Minnaard, ${ }^{1}$ Marthe T.C. Walvoort ${ }^{1, *}$

${ }^{1}$ Stratingh Institute for Chemistry, University of Groningen, Nijenborgh 7, 9747 AG Groningen, the Netherlands

${ }^{2}$ Interdepartmental Research Unit of Peptide and Protein Chemistry and Biology, Department of Chemistry "Ugo Schiff", University of Florence, Via della Lastruccia 13, 50019 Sesto Fiorentino, Italy

*Correspondence: m.t.c.walvoort@rug.nl

\section{Abstract}

Here we report a novel method of site-selective oxidation of glucose moieties on individual glycopeptides and on a mixture of tryptic glycopeptides. The organometallic catalyst $[(\text { neocuproine }) \mathrm{PdOAc}]_{2} \mathrm{OTf}_{2}$, that was previously shown to perform regioselective C3-oxidation of glucosides, was used in the scope of this work. The selectivity of the catalyst towards glucose and the sensitivity of specific amino acid residues to oxidation was explored by screening a select panel of glycopeptides in the oxidation reactions. We reveal that glucosylated peptides are more readily oxidized compared to galactosylated peptides, and Thr/Ser-oxidation is a concomitant side-reaction. The oxidation methodology was also applied to the complex mixture of tryptic glucopeptides that was generated from the fragment of Haemophilus influenzae adhesin glycoprotein. The resulting keto-group of the glucose was further transformed into an oxime functionality, which allows introduction of various groups of interest. The methodology outlined in this work will allow to perfrom late-stage modification of 
glucopeptides as well as selective oxidation and functionalization of tryptic glucopeptides for proteomics analysis.

\section{Introduction}

Carbohydrates form a diverse class of biomolecules with a myriad of roles in living cells. They form oligo- and polysaccharides or decorate proteins and lipids, thereby influencing the interactions and biological roles of these molecules. ${ }^{1}$ The presence of the carbohydrate moieties (glycans) confers a variety of properties to the resulting glycoconjugates, both biophysical (stability, solubility) and functional (transport, intra- and extracellular interactions). ${ }^{2}$ Importantly, aberrant glycosylation or altered levels and composition of the glycan structures are implicated in various diseases, including cancer. ${ }^{3,4}$ Bacterial cell surface glycoproteins frequently mediate motility, adherence, immune system evasion and biofilm formation, and as such constitute important virulence factors of pathogenic bacteria, and are considered interesting targets for antibiotic development. ${ }^{5,6}$

Interestingly, in recent years virulence-associated glycoproteins synthesized via unconventional glycosylation systems have been discovered and feature bacteria-specific rare sugars and glycosidic linkages. ${ }^{6}$ A notable example comprises the high molecular weight adhesin (HMWA) proteins from the Gram-negative human pathogen Haemophilus influenzae. ${ }^{7}$ Specifically, HMW1A is hyperglucosylated (i.e. modified with multiple glucose moieties) on 31 $\mathrm{N}$-glycosylation sites by the action of the associated $\mathrm{N}$-glycosyltransferase HMW1C in the cytoplasm. ${ }^{8}$ Extensive glucosylation is paramount for the stability of the adhesin protein and tethering to the cell membrane, where HMW1A is involved in adhering to the host cells during the initial stages of colonization. ${ }^{9}$ Studies of $H$. influenzae HMWA and other clinically relevant glycoproteins are complicated by the limitations of the available methods to selectively label these unusual glycoproteins. Glycoprotein synthesis is non-templated, often features microheterogeneity (i.e. multiple glycoforms of the same protein) and glycans are difficult to reliably manipulate on a genetic level. Therefore, methodologies that allow to unambiguously identify and enrich the glycoprotein of interest are highly valuable tools in glycobiology. In this 
regard, development of methods that allow the introduction of selective glycan modifications amenable for further manipulations are needed.

The metabolic oligosaccharide engineering (MOE) method, as pioneered by C. Bertozzi ${ }^{10,11}$ and W. Reutter, ${ }^{12}$ has introduced a multitude of possibilities for glycan manipulation. Through the development of unnatural sugar precursors bearing bioorthogonal reporter groups, metabolic pathways can be exploited to incorporate sugar analogues into the glycans of interest. The field of MOE has expanded over the recent years and now features multiple bioconjugation reactions, reporter tags and monosaccharide-based probes for profiling glycans of interest. ${ }^{13,14}$ Nonetheless, there are various challenges associated with MOE, in particular in the context of bacterial glycoproteins, such as the design and synthesis of unnatural monosaccharides, cell permeability of the probes and promiscuity of the metabolic pathways. Selective modification of a glycan or glycoconjugate of interest remains a challenging task which requires fine-tuning and adapting for each individual system of interest.

An alternative method to isolate glycoconjugates of interest is their derivatization with a bioorthogonal handle after their synthesis. This has been achieved enzymatically by oxidizing primary alcohols of the carbohydrate residues into their corresponding aldehydes (e.g. with oxidase enzymes) and chemically by oxidative cleavage of vicinal diols (e.g. with sodium periodate). The resulting carbonyl group can then be readily transformed into a hydrazone or oxime..$^{15}$ Enzymatic oxidation ${ }^{16-20}$ has been reported for galactose, mannose, GalNAc, and GlcNAc, and has been applied to the diversification of glycopeptide antibiotics and the introduction of amines and amides. ${ }^{21,22}$ In addition, oxidation and subsequent oxime/hydrazone formation can also be used as a strategy to introduce biotin or fluorophores (also on-cell). ${ }^{19-20}$ Similar to the MOE methodology, which relies on the enzyme specificities of the metabolic pathways, enzymatic oxidation is limited to the range of suitable substrates for the available oxidases.

Chemical oxidation of the glycan hydroxyl groups can be performed by sodium periodate, and this has been used extensively on galactose and sialic acid residues, also on glycopeptides. ${ }^{19,23-25}$ Although this type of oxidation is particularly effective for cis diols, the 
oxidation of trans diols such as O-GlcNAc-modified proteins can be achieved by using a high concentration of sodium periodate in combination with elevated temperatures. ${ }^{26} \mathrm{~A}$ drawback is that sodium periodate also acts on 1,2-aminoalcohols and rapidly cleaves $N$-terminal serine (Ser) and threonine (Thr) residues. ${ }^{23}$ In addition, sodium periodate may oxidize other residues in peptides as well, such as methionine, carbamidomethylated cysteine, and tryptophan, especially under the forcing conditions used in oxidative cleavage of vicinal trans-diols. ${ }^{23,26}$ Therefore, sodium periodate is not suitable to specifically modify glucosylated proteins and peptides.

In this work we describe the possibility to modify glucopeptides using site-selective palladium-catalyzed oxidation of unprotected carbohydrates. This method has been extensively studied by our group and the group of Waymouth, ${ }^{27-30}$ and has proven to be very useful for the selective oxidation of the $\mathrm{C} 3$ position in glucose-configured pyranosides. ${ }^{30,31}$ When applied to 1,4-linked glucans, the reaction is highly selective for the terminal residue, due to steric reasons (Figure 1). ${ }^{28}$ The resulting unprotected 3-keto glucosides can be used to prepare rare sugars and to introduce click handles and reactive groups, as was recently shown by Marinus et al. ${ }^{32}$ Although transition metal-catalyzed reactions, like with palladium, are widely used in synthetic chemistry, their use in biological settings is met with difficulties such as low conversions, the requirement of high temperatures or loss of protein. ${ }^{33-37}$ These catalysts have the tendency to coordinate with functional groups, such as amides and amines. For example, Ourailidou et al. observed during the oxidative Heck reaction that the binding of $\mathrm{Pd}(\mathrm{II})$ to the protein hampered the reaction by limiting the availability of the catalyst. ${ }^{38}$ This effect can be counteracted by selecting an appropriate ligand for the Pd catalyst, as was also recently reported by Cao et al. for the Suzuki-Miyaura reaction in cell lysates. ${ }^{39}$

Here we show that a palladium neocuproine catalyst system can be employed to selectively oxidize glucose residues in selected glucopeptide sequences, containing serine and threonine residues. The resulting keto function is used to attach useful tags and labels prior to proteomics experiments. The limits of this method are also described. Consequently, we add a new method to the toolbox for glycopeptide labeling. We expect that our method will be of use in 
the process of understanding the impact of specific glycoproteins in biological processes, with the glucosylation of HMWA adhesins as a prime example.

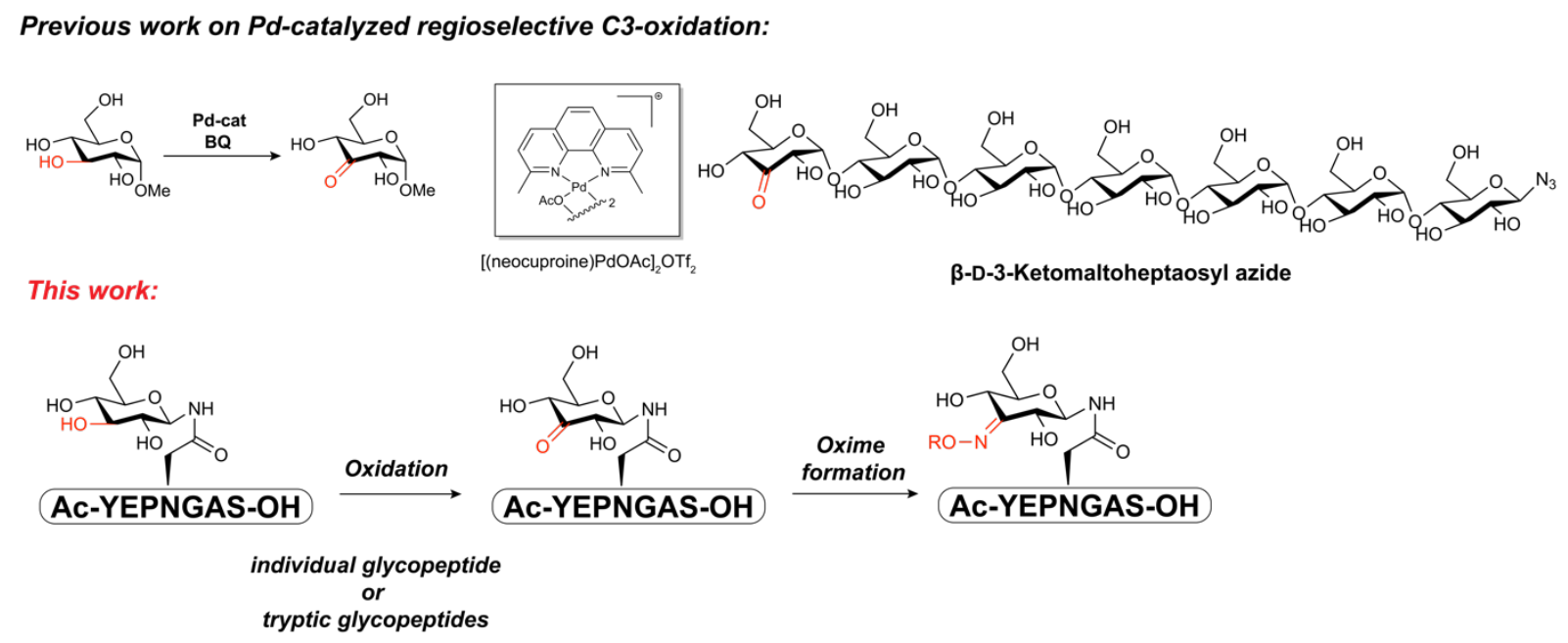

Figure 1. Previous and current scope of C3-selective oxidation of glucans and subsequent keto-group functionalization. $\mathrm{Pd}$-cat $=[(\text { neocuproine }) \mathrm{PdOAc}]_{2} \mathrm{OTf} f_{2}, \mathrm{BQ}=$ benzoquinone .

\section{Results and discussion}

Oxidation under dilute conditions. To translate our oxidation protocol, developed for preparative carbohydrate synthesis, to glycopeptides and ultimately glycoproteins, we first explored the lower limits of substrate concentration. The palladium-catalyzed oxidation of monosaccharides is generally performed in DMSO or methanol at relatively high concentrations $(0.1-0.3 \mathrm{M}){ }^{27,30,31}$ However, due to the low abundance of glycopeptides and glycoproteins in natural samples, these concentrations are not attainable, and our first goal was to screen for catalyst activity at dilute conditions. Both the $\alpha$ - and $\beta$-anomer of methyl Dglucopyranoside were subjected to the oxidation reaction in DMSO- $d_{6}$ using [(neocuproine)PdOAc $]_{2} \mathrm{OTf}_{2}$ as the catalyst and benzoquinone as the terminal oxidant. The reactions were performed in an NMR tube and monitored over time. As shown in the Table S1 (Supporting Information), full oxidation of the D-glucopyranosides was achieved at a concentration as low as $3 \mathrm{mM}$, and heating at $40{ }^{\circ} \mathrm{C}$ accelerated the oxidation. It has been 
shown earlier that the oxidation reaction is considerably accelerated in DMSO as the solvent. ${ }^{30,40}$ In line with this observation, the rate of oxidation decreased in DMSO/water mixtures, and increasing amounts of the Pd catalyst ( $0.1 \mathrm{eq} v s$ of $0.05 \mathrm{eq})$ were required to obtain full conversion in a mixture of $D_{2} \mathrm{O} / \mathrm{DMSO}-d_{6}(9 / 1 \mathrm{v} / \mathrm{v})$. Using qNMR it was revealed that oxidation of both the $\alpha$ - and $\beta$-anomer of methyl D-galactopyranoside led to full consumption of the monosaccharides (Table S2), however, significant amounts of degradation were observed for both substrates due to overoxidation and subsequent rearrangement, as observed before.$^{31}$ Interestingly, the dilute conditions as developed above significantly reduced the degradation after oxidation.

Chemoselective oxidation of model glycopeptides. In the next step, we selected the glucopeptide Ac-YEPN(GIc)GAS (1) to investigate the chemoselective oxidation of the asparagine-linked glucoside residue (Figure 2A). This glucopeptide was designed in such a way that it would show a reasonable solubility and the amino acid side chains would not interfere with the reaction. The workflow of the glycopeptide oxidation experiments is depicted in Figure $2 \mathrm{~B}$. The oxidation reactions were performed at $3 \mathrm{mM}(30 \mu \mathrm{L}$ total volume), and the reactions were monitored using reverse-phase liquid chromatography-mass spectrometry (RP-LCMS) (Figure 2C). Initial experiments showed that the dilute conditions used in the NMR experiments on monosaccharide oxidation did not yield any oxidized glucopeptide (Table 1, entry 1). However, increasing the amount of [(neocuproine)PdOAc $]_{2} \mathrm{OTf}_{2}$ to 0.5 eq (i.e. 1 equivalent of palladium, entry 2) led to the appearance of the peak corresponding to ketoproduct $2(\mathrm{~m} / \mathrm{z}=939)$. The mass corresponding to the deglycosylated product 3 was also detected $(\mathrm{m} / \mathrm{z}=779)$. Comparison of the RP-LCMS fragmentation profiles of peptides 1 and 2 proved that solely the glucose residue was oxidized (Figure 2D). To estimate the conversion, we compared the relative intensity of the peaks and corrected them for the isotope pattern, accounting for $88 \%$ conversion to 2 and $5 \%$ to 3 after 75 min reaction time (Figure 2C). For one sample, the conversion was also determined by calculating the relative ionization factor (Figure S1), ${ }^{41}$ which gave slightly higher conversions. Due to the minimal difference in the 
calculation methods, it was decided to calculate the conversions directly from the relative peak intensities.

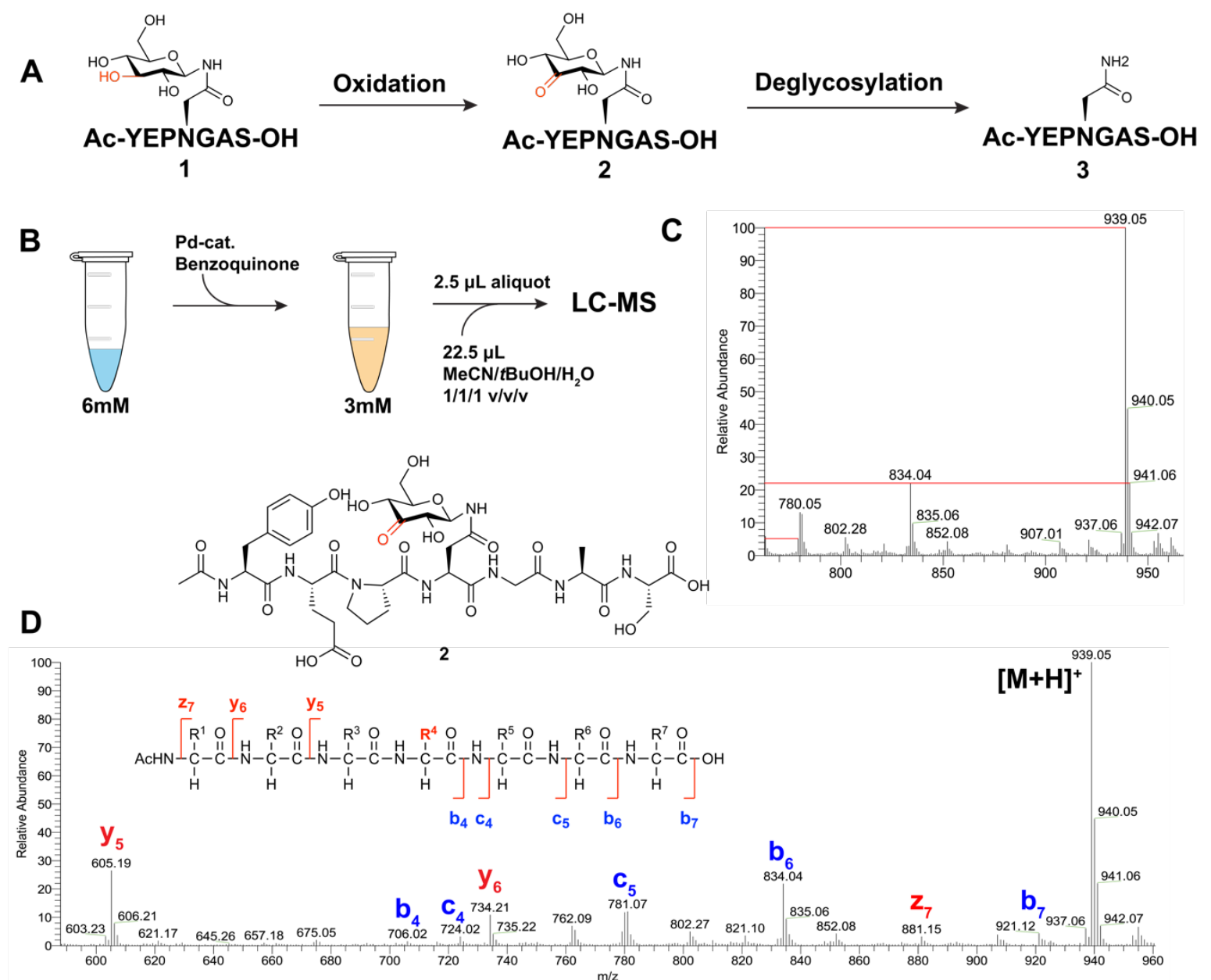

Figure 2. Workflow and analysis of the oxidation of glucopeptide 1. (A) Oxidation of glucopeptide 1 to keto-product 2 and deglycosylated product 3; (B) Setup of the oxidation reaction; (C) RP-LCMS analysis by comparison of the peak intensities. The mass values 941 , 939, and 779 correspond to peptides 1, 2, and 3 respectively; (D) MS/MS fragmentation of 2.

It was observed that longer reaction times led to increased formation of deglycosylated product 3. To understand the origin of this bond cleavage, we first investigated the stability of glucopeptide 1 in the presence of $\mathrm{Pd}(\mathrm{OAc})_{2}$ (entry 1, Table S3). Negligible formation of 3 was observed, suggesting that the deglycosylation reaction only occurs after oxidation, hence from 2. Addition of more Pd catalyst to a fully oxidized reaction mixture resulted in the increased 
formation of 3 (entry 2-3, Table S3). Moreover, removal of the Pd catalyst after the oxidation reaction by treatment with ammonium pyrrolidine dithiocarbamate (APDC) as a palladium scavenger suppressed the deglycosylation reaction (vide infra) ${ }^{42,43}$ We therefore conclude that the deglycosylation reaction is caused by the Pd catalyst, either via an $\mathrm{E} 1 \mathrm{cB}$ elimination or via overoxidation. In the first pathway, enolization of the carbonyl group is followed by $\mathrm{E} 1 \mathrm{cB}$ elimination of the anomeric substituent (see Figure $S 2$ for a proposed mechanism).$^{44,45}$ In the second proposed pathway, $\mathrm{C} 2-\mathrm{OH}$ is oxidized, which can then enolize and after formation of an hemiaminal hydrolysis takes place. A small signal corresponding to the overoxidized product $(\mathrm{m} / \mathrm{z}=939)$ was observed (Figure 2$)$.

Similar to the monosaccharide oxidation reactions, the oxidation of 1 occurs at a higher rate at elevated temperatures (compare entries 2 and 3, Table 1). A higher Pd catalyst loading gave a slightly faster conversion but resulted, as may be expected, also in further deglycosylation (entries 4 and 5). Oxidation reactions in different solvent mixtures revealed that oxidation of 1 in DMSO- $d_{6} / \mathrm{D}_{2} \mathrm{O}$ mixture (entries 6-8) is possible, however, longer reaction times and more $\mathrm{Pd}$ catalyst are required. These results suggest that this method may have potential for the oxidation of glycoproteins in aqueous media, a perspective that will be studied in the near future.

To assess whether the preference for glucose over galactose, as observed in monosaccharides oxidation reactions, ${ }^{31}$ was also present in the corresponding glycopeptides, we investigated the oxidation of the glycopeptide $\mathbf{S 9}$ containing a galactose moiety (Table S4). The oxidation occurred considerably slower and more Pd catalyst was required compared to the corresponding glucopeptide 1 . This suggests that it is may be possible to oxidize glucose residues in the presence of galactose residues in a glycopeptide. 
Table 1. C-3 Oxidation of Glucose in a Glycopeptide ${ }^{[a]}$

\begin{tabular}{|c|c|c|c|c|}
\hline$\#$ & $\begin{array}{l}\text { Temp. } \\
\left({ }^{\circ} \mathrm{C}\right)\end{array}$ & Solvent & $\begin{array}{l}\text { Pd-cat. } \\
\text { (eq) }\end{array}$ & $\begin{array}{c}\text { Conversion (\%) } \\
\qquad \mathbf{2 / 3}\end{array}$ \\
\hline 1 & 40 & DMSO- $d_{6}$ & 0.05 & ON: $0 / 0$ \\
\hline 2 & 40 & DMSO- $d_{6}$ & 0.5 & $75 \min : 88 / 5$ \\
\hline 3 & 22 & DMSO- $d_{6}$ & 0.5 & $\begin{array}{c}150 \text { min: } 69 / 5 \\
\text { ON: } 81 / 15\end{array}$ \\
\hline 4 & 40 & DMSO- $d_{6}$ & 1 & $\begin{array}{c}75 \text { min: } 85 / 9 \\
\text { ON: } 35 / 58\end{array}$ \\
\hline $5^{[b]}$ & 40 & DMSO- $d_{6}$ & 3 & $\begin{array}{c}75 \text { min: } 87 / 12 \\
\text { ON: } 10 / 90\end{array}$ \\
\hline 6 & 40 & DMSO- $d_{6} / \mathrm{D}_{2} \mathrm{O}: 9 / 1 \mathrm{v} / \mathrm{v}$ & 1 & $\begin{array}{l}75 \min : 82 / 6 \\
\text { ON: } 51 / 45\end{array}$ \\
\hline $7^{[\mathrm{c}]}$ & 40 & DMSO- $d_{6} / \mathrm{D}_{2} \mathrm{O}: 1 / 1 \mathrm{v} / \mathrm{v}$ & 0.5 & ON: $37 / 29$ \\
\hline $8^{[\mathrm{b}]}$ & 40 & DMSO- $d_{6} / \mathrm{D}_{2} \mathrm{O}: 1 / 1 \mathrm{v} / \mathrm{v}$ & 3 & $\begin{array}{l}7 \mathrm{~h}: 54 / 19 \\
\text { ON: } 14 / 83\end{array}$ \\
\hline
\end{tabular}

${ }^{[a]}$ Reactions were performed at $3 \mathrm{mM}$ concentration in the presence of 3 eq benzoquinone. RPLCMS samples were prepared by $10 x$ dilution with $\mathrm{CH}_{3} \mathrm{CN} / \mathrm{tBuOH} / \mathrm{H}_{2} \mathrm{O} 1 / 1 / 1 \mathrm{v} / \mathrm{v} / \mathrm{v}$ to obtain a $0.3 \mathrm{mM}$ concentration. Samples of entries with more than 1 eq of $\mathrm{Pd}$ catalyst were treated with APDC before RP-LCMS analysis. Conversions were calculated by comparison of the relative ionization intensities of the corresponding molecular ions. ${ }^{[b]}$ No benzoquinone was added. ${ }^{[c]} \mathrm{A}$ DMSO- $d_{6} / \mathrm{D}_{2} \mathrm{O}$ mixture $(1 / 9 \mathrm{v} / \mathrm{v})$ resulted in slower oxidation.

Oxidation of complex glycopeptides. Next, we selected two peptide sequences $\mathbf{5}$ and $\mathbf{6}$ (Table 2) of the bacterial high molecular weight adhesin protein HMW1A, known to be glucosylated on specific asparagine residues in Haemophilus influenzae. ${ }^{8}$ Compared to the non-glycosylated control peptide $\mathbf{4}$, glucopeptides 5 and $\mathbf{6}$ both contain one Glc moiety, in 
addition to serine, threonine, and lysine residues. These residues were selected to pose two challenges. The hydroxyl functions of serine and threonine side chains might be oxidized by the catalyst. The lysine residue might block the oxidation reaction, coordinating palladium and thus poisoning the catalyst. ${ }^{38}$ To get an indication of the sensitivity of threonine towards oxidation by the $\mathrm{Pd}$ catalyst, we treated peptide 4 (containing two Thr) with one eq of $\mathrm{Pd}$ catalyst (Table 2, entry 1 ). It turned out that the secondary hydroxyl group on Thr was indeed oxidized, with the singly oxidized compound as the major product compared to the twice oxidized product. The mass fragmentation pattern of this oxidized peptide indicates that the Cterminal Thr is oxidized first. Longer reaction times led to the formation of degradation products, probably as a result of overoxidation, in which the C-terminal Thr or the peptide fragment Thr-Ile-Thr (TIT) is split off. Proposed mechanisms are reported in Figure S3, wherein it is shown that either overoxidation can take place or, after oxidation of Thr, tautomerization to the corresponding enol occurs. Subsequent imine formation followed by water addition forms a hemiaminal product, which is readily hydrolyzed. This side-reaction has also been observed by Bose et al. in the synthesis of $\beta$-lactams ${ }^{46}$ and by Abeysinghe et al. when using o-iodoxybenzoic acid as an oxidizing agent. ${ }^{47}$

NMR experiments with $\mathrm{N}$-acetyl-threonine confirmed that the $\mathrm{Pd}$ catalyst oxidized the secondary hydroxyl group in threonine, although at least 0.5 eq catalyst was required to obtain full conversion (entry 2, Table S5). Encouragingly, when $\mathrm{N}$-acetyl-threonine and methyl Dglucopyranoside were combined in equal amounts in a competition oxidation experiment, a strong preference for glucose oxidation was observed at earlier time points (entry 3, Table S5). 
Table 2. Oxidation of glucose in the presence of threonines and lysine $e^{[a]}$

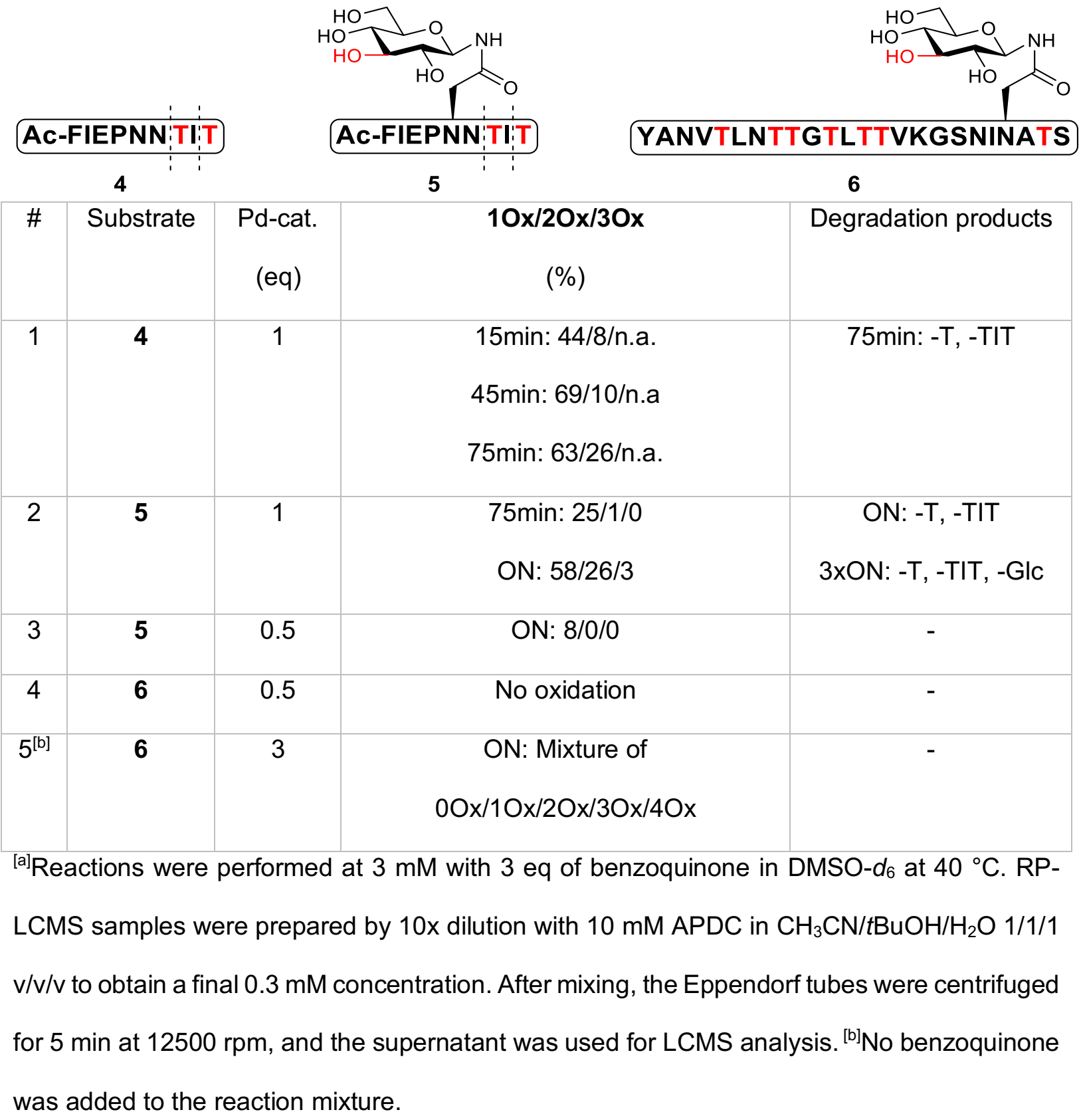

Therefore, we continued the oxidation experiments with glycopeptide $\mathbf{5}$. The use of 1 eq of Pd catalyst led to $58 \%$ of a mono-oxidized product (Table 2, entry 2). Fragmentation showed that the glucose moiety is oxidized (based on the $b_{6}$ fragment ion, peptide $\mathbf{5}$ spectra in the Supporting information). Unfortunately, we were not able to determine the ratio between the oxidized glucose and threonine. Similarly to the oxidation of glucopeptide $\mathbf{1}$ and peptide $\mathbf{4}$, degradation products were formed upon prolonged reaction times. ${ }^{48}$ Treatment with APDC and 
charcoal proved instrumental in facilitating the LC-MS analysis of 5 by removing overlapping ligand peaks from the chromatogram. In an attempt to suppress these side-reactions, the amount of catalyst was reduced to 0.5 eq (Table 2 , entry 3 ), which resulted in a low conversion $(8 \%)$ to the mono-oxidized product, which slowly increased over time.

It was not possible to assign the oxidation site due to the presence of the different oxidationprone residues. Therefore, we decided to perform a set of competition experiments to investigate the kinetics of threonine and carbohydrate oxidation reaction. To this end, we combined glucopeptide 1 with control peptide 4, and used RP-LCMS to monitor oxidation progress (Table S6). In the presence of 0.5 eq $\mathrm{Pd}$ catalyst and $3 \mathrm{eq}$ of benzoquinone, glucopeptide 1 was preferentially oxidized, albeit at a lower rate due to the presence of the threonine-containing peptide 4 . Increasing the amount of $\mathbf{4}$ slowed down the oxidation of $\mathbf{1}$ even more, resulting in a decrease in turnover number (TON) by more than $50 \%$. We hypothesize that the catalyst can form a bidentate chelate with threonine via the hydroxyl group and the carbonyl amide in the peptide backbone. As such, threonine residues compete for chelation with the Pd with the vicinal diol units in glucose and thereby slow down the oxidation of glucopeptide 1. Notably, when a similar competition experiment was performed on an equal mixture of peptide 4 and galactosylated peptide S9, with the same peptide sequence as glucopeptide 1, no oxidation of either galactose or threonine was observed. This suggests that galactose, while being a poor substrate for oxidation, chelates well with the Pd catalyst and thereby inhibits threonine oxidation. Overall, these competition experiments highlight again the strong preference of the catalyst to oxidize gluco-configured moieties.

Finally, glycopeptide 6 was subjected to the oxidation conditions (Table 2, entries 4 and 5). Despite the presence of an $N$-terminal amine and the amine of the lysine side chain, oxidation was observed with an increased catalyst loading (3 eq). Similar to glycopeptide $\mathbf{5}$, however, the multiple oxidation-sensitive positions hampered the assignment of the oxidation products. Nonetheless, this shows that the oxidation reaction is possible in the presence of amines, setting the stage for even more complex peptides and mixtures (vide infra). 
Oxidation of tryptic glucopeptides. Encouraged by the successful oxidation of individual glycopeptides, we next used our methodology in the oxidation of a complex tryptic digest of a glucoprotein. For this we used the glucosylated C-terminal fragment of the high molecular weight adhesin (HMWA) protein from Haemophilus influenzae. The hyperglucosylated Cterminal fragment of HMWA (termed Glc-HMW1ct) was produced via co-expression with its cognate $N$-glycosyltransferase, as described previously. ${ }^{49}$ Glc-HMW1ct was purified using anion exchange chromatography and intact protein mass-spectrometry revealed the presence of 8-9 glucose moieties per adhesin protein. The glycoprotein sample was digested with trypsin to obtain shorter glycopeptides. There is a considerable degree of diversity among the peptide fragments with regard to length, number of glucose residues per peptide and number of Ser and Thr residues (Figure 3, Figure S4).

The workflow for the oxidation reactions on the tryptic glycopeptides is depicted in Figure 3. After tryptic digestion, the glucopeptides were purified via solid phase extraction (SPE) to remove buffer salts that could interfere with the reaction, and were dried under vacuum. The resulting residue was reconstituted in DMSO to prepare a stock solution for the oxidation reaction. The oxidation was performed at $2.8 \mathrm{mM}$ concentration of glycopeptides (molarity based on glucose and calculated based on the input protein amount) at $40{ }^{\circ} \mathrm{C}$ with varying amounts of the $\mathrm{Pd}$ catalyst $(0.5,1,5$ and 10 eq calculated relative to Glc residues). 


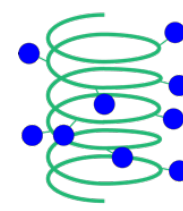

Glc-HMW1ct avg 8-9GIc
1. Tryptic digest

2. SPE

Hexose on Asn (+162 Da)

- Oxidized hexose on Asn (+160 Da)

-Oxime modification

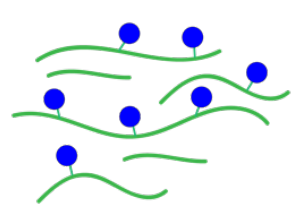

mixture of tryptic (gluco)peptides

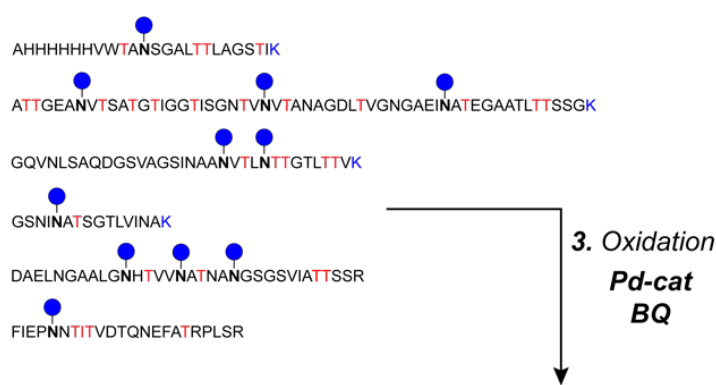

4. Oxidized glucopeptides

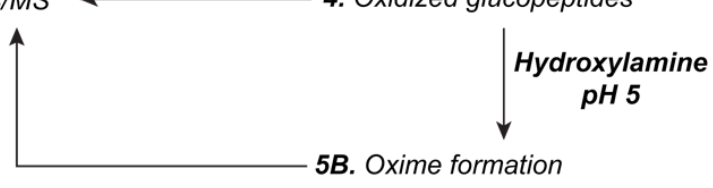

Figure 3. Workflow of the oxidation reaction and oxime formation on tryptic glycopeptides. (SPE = solid-phase extraction).

The highest Pd catalyst loadings (5 eq and 10 eq) led to a substantial decrease in general peptide levels (Figure S5A). In contrast, 0.5 and 1 eq of Pd did not result in significant changes in peptide levels compared to the non-treated control sample (Figure S5B) and therefore the oxidation of the tryptic glycopeptides was performed with either 0.5 eq or 1 eq of Pd catalyst. The reactions were analyzed after 8 and $24 \mathrm{~h}$ by diluting reaction aliquots with an aqueous solution of the Pd catalyst scavenger APDC (vide supra). Following another SPE purification to remove traces of DMSO, ligand, and $B Q$, the aliquots were subjected to RP-LCMS/MS analysis. Several glucopeptides were identified in the oxidized and the control samples and these glucopeptides could be assigned to one of the three categories: non-oxidized glucopeptides (so-called hexose fraction, "Hex"), glucopeptides containing both glucose and oxidized glucose moieties (mixed fraction, "Mix") and glucopeptides in which all glucose residues were fully oxidized (oxidized fraction, "OxHex"). Examples are presented in Figure 4A. The peak areas of the peptides were summed and plotted as depicted in Figure 4B, C. 

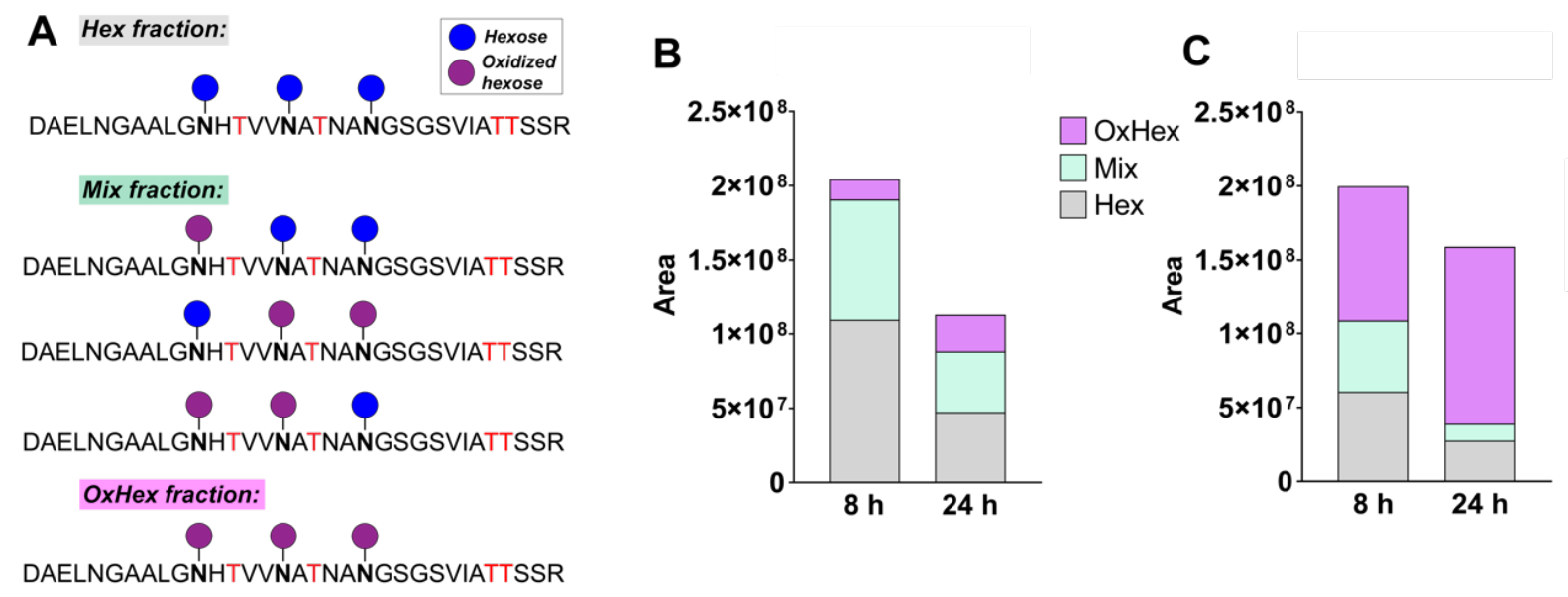

Figure 4. (A) Examples of the peptide selected for hexose (Hex), mixed (Mix), or oxidized (OxHex) fractions. Oxidation of tryptic glycopeptides from Glc-HMW1ct after $8 \mathrm{~h}$ and $24 \mathrm{~h}$ oxidation reaction with 0.5 eq $(B)$ and 1 eq $(C)$ of $P d$ catalyst. The $Y$-axis represents the peptide peak areas of the glycopeptides detected in LC-MS/MS. Representative peptide spectra can be found in the Supporting information.

As shown in Figure 4B, after $8 \mathrm{~h}$ with 0.5 eq of $\mathrm{Pd}$ catalyst already significant oxidation of glycopeptides was observed. After $24 \mathrm{~h}$ of oxidation with 0.5 eq of Pd catalyst, the mixture contained oxidized glycopeptides as the major fraction, but the total level of peptides decreased. For the oxidation reaction with 1 eq Pd catalyst (Figure 4C), a fully oxidized fraction was already dominant after $8 \mathrm{~h}$, and longer reaction times led to an even higher extent of oxidation. Therefore, 1 eq of Pd catalyst and 8-24 h of incubation were found to be the optimal oxidation conditions to afford high levels of oxidation without compromising the peptide levels. Differences in the protein sequence coverage can be found in the Supplementary Information (Table S7 and protein sequence coverage exports). A certain degree of Thr (and Ser) oxidation was detected alongside glucose oxidation, however, it was difficult to reliably quantify as it typically coincided with other modifications in the peptides.

Introduction of (affinity) tags via oxime formation. The selective introduction of affinity or fluorescence labels to glycopeptides is both highly valuable and difficult to achieve. ${ }^{50-52}$ The 
tag allows to identify, pulldown, enrich and profile glycoproteins of interest that are typically present in low amounts in biological samples. For the purpose of expanding the oxidation methodology presented in this work towards biological applications, we investigated whether the oxidized mixture of glycopeptides can be further modified with a tag of interest via oxime ligation (Figure 5).

First, the process of oxime formation on pure glycopeptide 1 was investigated. After the oxidation reaction, a sample was diluted with a $100 \mathrm{mM} \mathrm{NaOAc/AcOH}$ buffer $(\mathrm{pH}=5)$ and subjected to a treatment with APDC to remove Pd species and prevent deglycosylation. After the addition of hydroxylamine, the reaction mixture was kept at $40{ }^{\circ} \mathrm{C}$ overnight. Under these conditions, oxime 7 was formed in $52 \%$, which could not be increased by a longer reaction time or more hydroxylamine. It should be noted that only a slight increase of $5-10 \%$ of deglycosylated peptide 3 was observed overnight.

Encouraged by this result, 3-keto peptide derivative 2 was treated with a biotinylated alkoxyamine derivative. LC-MS analysis revealed the formation of oxime 8 , but we were unable to accurately estimate the conversion due to the differences in ionization of the peptides.

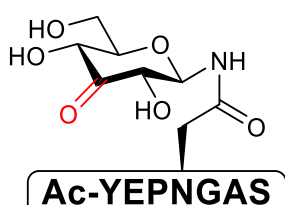

2

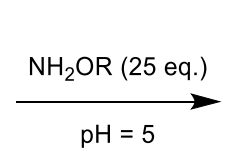

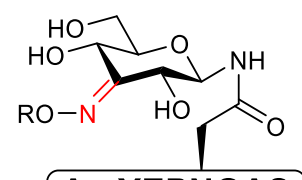

Ac-YEPNGAS
$7 \mathrm{R}=\mathrm{H}$ (52\% conversion)

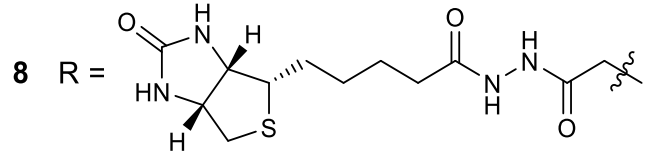

Figure 5. Transforming the 3-keto Glc into an oxime.

Next, we applied these reaction conditions to the mixture of oxidized tryptic peptides. The results after incubation with hydroxylamine are presented in Figure 6. Compared to the oxidation reaction, two additional fractions were identified: peptides that contained both an oxime and hexose/oxidized hexose modifications (mixed oxime fraction) and peptides that contained only oxime modification (oxime fraction). To simplify data analysis, mixed and fully oxidized peptides were grouped together as "OxHex" fraction, while peptides bearing at least 
one oxime modification were designated as "Oxime" fraction (examples are shown in Figure 6A). As is shown in Figure 6B, oxime formation from the 0.5 eq reaction aliquot after $8 \mathrm{~h}$ (and $24 \mathrm{~h}$ ) of oxidation resulted in only small amounts of oxime product. Importantly, the aliquot after $24 \mathrm{~h}$ reaction with 1 eq of catalyst contained a large portion of hydroxylamine oxime product (Figure 6C). When alkoxyamine-biotin was used, a small amount of oxime product was detected in all samples (Figure 6D and E) with 0.5 eq $24 \mathrm{~h}$ aliquot containing the most significant oxime fraction.

Interestingly, whereas multiple tryptic glycopeptides were identified in the non-treated adhesin protein sample (Figure S4), reaction aliquots from the oxidation and oxime formation typically contained three major glycopeptides (Table S8). This "enrichment" may reflect the preference for length and amino acid composition, or a higher stability of certain glucopeptides under the reaction conditions for oxidation and oxime formation.

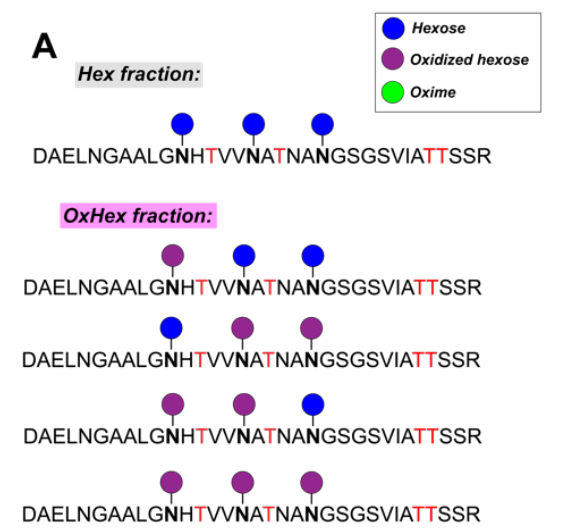

\section{Hydroxylamine oxime:}
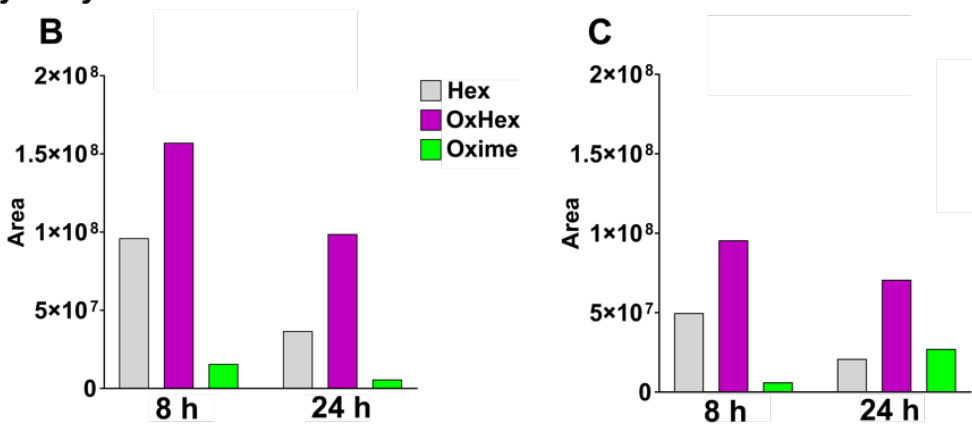

Biotin oxime:
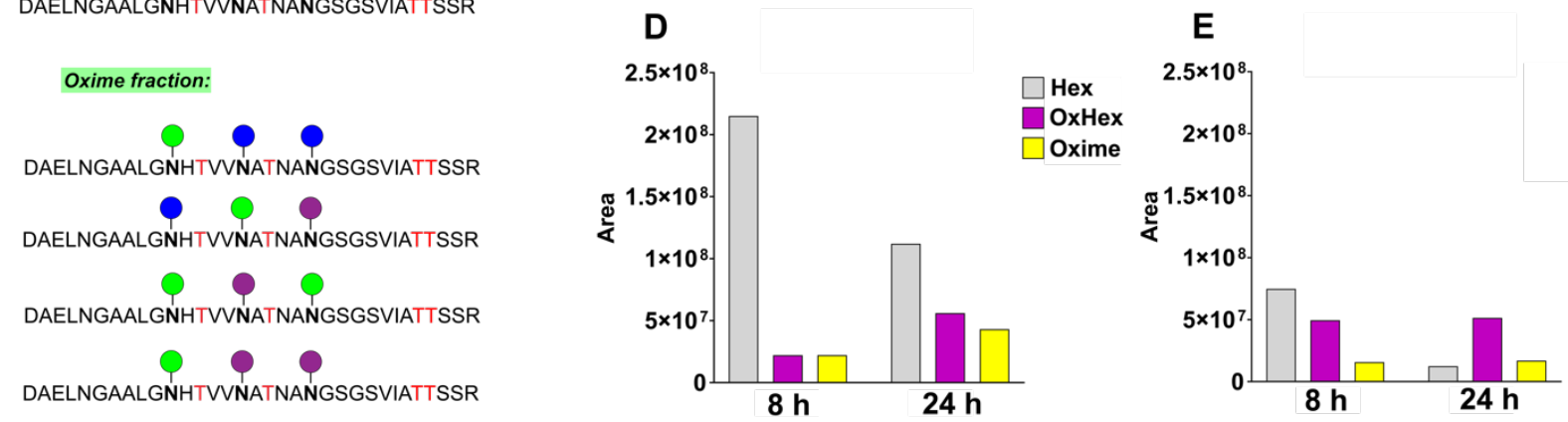

Figure 6. (A) Examples of the peptides selected for hexose (Hex), oxidized hexose (OxHex), or oxime (Oxime) fractions. Oxime formation with hydroxylamine after oxidation with 0.5 eq (B) and 1 eq $(C)$ of Pd catalyst. Biotin oxime formation after oxidation with 0.5 eq (D) and 1 eq (E) of Pd catalyst. Representative peptide spectra can be found in the Supporting information. 


\section{Conclusions}

Site-selective Pd-catalyzed oxidation, developed for unprotected carbohydrates, has successfully been applied to glycopeptides. This is a significant step forward, as the tendency of transition metals, including palladium, to chelate to peptides and proteins is an inherent bottleneck for such an approach. The conditions for the oxidation reaction were optimized for $3 \mathrm{mM}$ substrate concentration, to make these suitable for glycopeptides and glycoproteins that are typically obtained in low amounts. Oxidation of single glycopeptides demonstrates that it may be possible to selectively perform the oxidation of a glucose moiety in the presence of a galactose moiety, since the latter is far less reactive and requires higher catalyst loadings. Similar to sodium periodate oxidation, the Pd-catalyzed oxidation has some limitations, as Thr residues were found to be oxidized, and $\mathrm{Pd}$-mediated deglycosylation was observed. Fortunately, deglycosylation can successfully be suppressed by tuning the reaction conditions and treatment with a Pd scavenger.

In addition, we have expanded the substrate scope of the Pd-catalyzed oxidation to tryptic glucopeptides from the immunologically relevant $H$. influenzae adhesin protein. Sufficient levels of glucose oxidation were obtained to further exploit the introduced keto group in a bioconjugation reaction with hydroxylamine to form the desired oxime. Based on this, the widely used affinity tag biotin was incorporated in low to moderate levels. Taken together, Pdcatalyzed oxidation allows the selective modification of glucoproteins and this observation can now be further developed for proteomics identification and pulldown.

\section{Acknowledgements}

We thank Gemma Sturt and Dr. Chiara Testa for technical assistance in the glycopeptide synthesis, and Dr. Franz Ho for his input in the proteomics analysis. This work was financially supported by the Dutch Organization for Scientific Research (VENI 722.016.006) and the European Union through the Rosalind Franklin Fellowship COFUND project 60021 (both to M.T.C.W.). Fondazione Ente Cassa di Risparmio di Firenze (Italy) (grant n. 2014/0306) is gratefully acknowledged for supporting the equipment to PeptLab of the University of Florence. 


\section{References}

1. Varki, A. Biological roles of glycans. Glycobiology 27, 3-49 (2017).

2. Ohtsubo, K., Marth, J. D. Glycosylation in Cellular Mechanisms of Health and Disease. Cell 126, 855-867 (2006).

3. Pinho, S. S., Reis, C. A. Glycosylation in cancer: mechanisms and clinical implications. Nat. Rev. Cancer 15, 540-555 (2015).

4. Reily, C., Stewart, T. J., Renfrow, M. B., Novak, J. Glycosylation in health and disease. Nat. Rev. Nephrol. 15, 346-366 (2019).

5. Valguarnera, E., Kinsella, R. L., Feldman, M. F. Sugar and Spice Make Bacteria Not Nice: Protein Glycosylation and Its Influence in Pathogenesis. J. Mol. Biol. 428, 3206-3220 (2016).

6. Fulton, K. M., Smith, J. C., Twine, S. M. Clinical applications of bacterial glycoproteins. Expert Rev. Proteomics 13, 345-353 (2016).

7. St Geme III, J. W., Falkow, S., Barenkamp, S. J. High-molecular-weight proteins of nontypable Haemophilus influenzae mediate attachment to human epithelial cells. Proc. Natl. Acad. Sci. 90, 2875-2879 (1993).

8. Grass, S., Lichti, C. F., Townsend, R. R., Gross, J., St. Geme III, J. W. The Haemophilus influenzae HMW1C Protein Is a Glycosyltransferase That Transfers Hexose Residues to Asparagine Sites in the HMW1 Adhesin. PLoS Pathog. 6, e1000919 (2010).

9. Grass, S. Buscher, A. Z., Swords, W. E., Apicella, M. A., Barenkamp, S. J., Ozchlewski, N., St. Geme III, J. W. The Haemophilus influenzae HMW1 adhesin is glycosylated in a process that requires HMW1C and phosphoglucomutase, an enzyme involved in lipooligosaccharide biosynthesis. Mol. Microbiol. 48, 737-751 (2003).

10. Saxon, E., Bertozzi, C. R. Cell Surface Engineering by a Modified Staudinger Reaction. Science 287, 2007-2010 (2000).

11. Mahal, L. K., Yarema, K. J., Bertozzi, C. R. Engineering Chemical Reactivity on Cell Surfaces Through Oligosaccharide Biosynthesis. Science 276, 1125-1128 (1997).

12. Kayser, H., Zeitler, R., Kannicht, C., Grunow, D., Nuck, R., Reutter, W. Biosynthesis of a nonphysiological sialic acid in different rat organs, using $\mathrm{N}$-propanoyl-D-hexosamines as precursors. J. Biol. Chem. 267, 16934-16938 (1992).

13. Sminia, T. J., Zuilhof, H., Wennekes, T. Getting a grip on glycans: A current overview of the metabolic oligosaccharide engineering toolbox. Carbohydr. Res. 435, 121-141 (2016).

14. Gilormini, P.-A., Batt, A. R., Pratt, M. R., Biot, C. Asking more from metabolic oligosaccharide engineering. Chem. Sci. 9, 7585-7595 (2018).

15. Palaniappan, K. K., Bertozzi, C. R. Chemical Glycoproteomics. Chem. Rev. 116, 14277-14306 (2016).

16. Hui, J., Bao, L., Li, S., Zhang, Y., Feng, Y., Ding, L., Ju, H. Localized Chemical Remodeling for Live Cell Imaging of Protein-Specific Glycoform. Angew. Chemie 129, 8251-8255 (2017).

17. Ramya, T. N. C., Weerapana, E., Cravatt, B. F., Paulson, J. C. Glycoproteomics enabled by tagging sialic acid- or galactose-terminated glycans. Glycobiology 23, 211-221 (2013).

18. Taga, Y., Kusubata, M., Ogawa-Goto, K., Hattori, S. Development of a novel method for analyzing collagen O-glycosylations by hydrazide chemistry. Mol. Cell. Proteomics 11, (2012).

19. McCombs, J. E., Kohler, J. J. Pneumococcal Neuraminidase Substrates Identified through Comparative Proteomics Enabled by Chemoselective Labeling. Bioconjug. Chem. 27, 1013$1022(2016)$.

20. Whitworth, G. E., Imperiali, B. Selective biochemical labeling of Campylobacter jejuni cellsurface glycoconjugates. Glycobiology 25, 756-766 (2015).

21. Li, T.-L., Liu, Y.-C., Lyu, S.-Y. Combining biocatalysis and chemoselective chemistries for glycopeptide antibiotics modification. Curr. Opin. Chem. Biol. 16, 170-178 (2012).

22. Marschall, E., Cryle, M. J., Tailhades, J. Biological, chemical, and biochemical strategies for modifying glycopeptide antibiotics. J. Biol. Chem. 294, 18769-18783 (2019).

23. Huang, J., Qin, H., Sun, Z., Huang, G., Mao, J., Cheng, K., Zhang, Z., Wan, H., Yao, Y., Dong, J., Zhu, J., Wang, F., Ye, M., Zou, H. A peptide N-terminal protection strategy for comprehensive glycoproteome analysis using hydrazide chemistry based method. Sci. Rep. 5, 10164 (2015).

24. Huang, J., Wan, H., Yao, Y., Li, J., Cheng, K., Mao, J., Chen, J., Wang, Y., Qin, H., Zhang, W., Ye, M., Zou, H. Highly Efficient Release of Glycopeptides from Hydrazide Beads by Hydroxylamine Assisted PNGase F Deglycosylation for N-Glycoproteome Analysis. Anal. Chem. 87, 10199-10204 (2015).

25. Ramya, T. N. C., Weerapana, E., Cravatt, B. F., Paulson, J. C. Glycoproteomics enabled by tagging sialic acid- or galactose-terminated glycans. Glycobiology 23, 211-221 (2013). 
26. Klement, E., Lipinszki, Z., Kupihár, Z., Udvardy, A., Medzihradszky, K. F. Enrichment of OGIcNAc Modified Proteins by the Periodate Oxidation-Hydrazide Resin Capture Approach. J. Proteome Res. 9, 2200-2206 (2010).

27. Chung, K., Waymouth, R. M. Selective Catalytic Oxidation of Unprotected Carbohydrates. ACS Catal. 6, 4653-4659 (2016).

28. Eisink, N. N. H. M., Lohse, J., Witte, M. D., Minnaard, A. J. Regioselective oxidation of unprotected 1,4 linked glucans. Org. Biomol. Chem. 14, 4859-4864 (2016).

29. Wan, I. C., Hamlin, T. A., Eisink, N. N. H. M., Marinus, M., de Boer, C., Vis, C. A. Codée, J. D. C., Witte, M. D., Minnaard, A. J., Bickelhaupt, F. M. On the Origin of Regioselectivity in Palladium-Catalyzed Oxidation of Glucosides. European J. Org. Chem. 632-636 (2021).

30. Jäger, M., Hartmann, M., de Vries, J. G., Minnaard, A. J. Catalytic Regioselective Oxidation of Glycosides. Angew. Chemie Int. Ed. 52, 7809-7812 (2013).

31. Eisink, N. N. H. M., Witte, M. D., Minnaard, A. J. Regioselective Carbohydrate Oxidations: A Nuclear Magnetic Resonance (NMR) Study on Selectivity, Rate, and Side-Product Formation. ACS Catal. 7, 1438-1445 (2017).

32. Marinus, N., Tahiri, N., Duca, M., Mouthaan, L. M. C. M., Bianca, S., van den Noort, M., Poolman, B., Witte, M. D., Minnaard, A. J. Stereoselective Protection-Free Modification of 3Keto-saccharides. Org. Lett. 22, 5622-5626 (2020).

33. Chalker, J. M., Bernardes, G. J. L., Davis, B. G. A "Tag-and-Modify" Approach to Site-Selective Protein Modification. Acc. Chem. Res. 44, 730-741 (2011).

34. Kodama, K., Fukuzawa, S., Nakayama, H., Sakamoto, K., Kigawa, T., Yabuki, T., Matsuda, N., Shirouzu, M., Takio, K., Yokoyama, S., Tachibana, K. Site-Specific Functionalization of Proteins by Organopalladium Reactions. ChemBioChem 8, 232-238 (2007).

35. Kodama, K., Fukuzawa, S., Sakamoto, K., Nakayama, H., Kigawa, T., Yabuki, T., Matsuda, N., Shirouzu, M., Takio, K., Tachibana, K., Yokoyama, S. A New Protein Engineering Approach Combining Chemistry and Biology, Part I; Site-Specific Incorporation of 4-lodo-L-phenylalanine in vitro by Using Misacylated Suppressor tRNAPhe. ChemBioChem 7, 1577-1581 (2006).

36. Isenegger, P. G., Davis, B. G. Concepts of Catalysis in Site-Selective Protein Modifications. J. Am. Chem. Soc. 141, 8005-8013 (2019).

37. Brustad, E., Bushey, M. L., Lee, J. W., Groff, D., Liu, W., Schultz, P. G. A Genetically Encoded Boronate-Containing Amino Acid. Angew. Chemie Int. Ed. 47, 8220-8223 (2008).

38. Ourailidou, M. E., Dockerty, P., Witte, M., Poelarends, G. J., Dekker, F. J. Metabolic alkene labeling and in vitro detection of histone acylation via the aqueous oxidative Heck reaction. Org. Biomol. Chem. 13, 3648-3653 (2015).

39. Cao, J., Boatner, L. M., Desai, H. S., Burton, N. R., Armenta, E., Chan, N. J., Castellón, J. O., Backus, K. M. Multiplexed CuAAC Suzuki-Miyaura Labeling for Tandem Activity-Based Chemoproteomic Profiling. Anal. Chem. 93, 2610-2618 (2021).

40. Painter, R. M., Pearson, D. M., Waymouth, R. M. Selective Catalytic Oxidation of Glycerol to Dihydroxyacetone. Angew. Chemie Int. Ed. 49, 9456-9459 (2010).

41. Kightlinger, W., Lin, L., Rosztoczy, M., Li, W., DeLisa, M. P., Mrksich, M., Jewett, M. C. Design of glycosylation sites by rapid synthesis and analysis of glycosyltransferases article. Nat. Chem. Biol. 14, 627-635 (2018).

42. Gallagher, W. P., Vo, A. Dithiocarbamates: Reagents for the Removal of Transition Metals from Organic Reaction Media. Org. Process Res. Dev. 19, 1369-1373 (2015).

43. de Bruijn, A. D., Roelfes, G. Catalytic Modification of Dehydroalanine in Peptides and Proteins by Palladium-Mediated Cross-Coupling. Chem. - A Eur. J. 24, 12728-12733 (2018).

44. Kunz, H. \& Weißmüller, J. Synthese von 2-Acyloxy- und 2-Alkyloxy-3-ketoglycalen und ihren Bromaddukten. Liebigs Ann. der Chemie 1561-1575 (1983).

45. Lichtenthaler, F. W., Jarglis, P. Sugar enolones, XII. Peroxidation of pyranose-derived enol esters: An efficacious synthesis of peracetylhexosuloses and their conversion into $y$-pyronesvia 3,2-enolones. Chem. Ber. 113, 489-510 (1980).

46. Bose, A. K., Manhas, M. S., Vincent, J. E., Gala, K., Fernandez, I. F. Studies on lactams. Part 65. N-Unsubstituted .beta.-lactams from .beta.-hydroxy-.alpha.-amino acids. Facile preparation of intermediates for isocephalosporins. J. Org. Chem. 47, 4075-4081 (1982).

47. Abeysinghe, P. M., Han, Y., Harding, M. M. Oxidation of threonine residues with IBX reagents. Tetrahedron Lett. 50, 2601-2604 (2009).

48. Li, C., Tebo, A. \& Gautier, A. Fluorogenic Labeling Strategies for Biological Imaging. Int. J. Mol. Sci. 18, 1473 (2017)

49. Walvoort, M. T. C., Testa, C., Eilam, R., Aharoni, R., Nuti, F., Rossi, G., Real-Fernandez, F., Lanzillo, R., Morra, V. B., Lolli, F., Rovero, P., Imperiali, B., Papini, A. M. Antibodies from 
multiple sclerosis patients preferentially recognize hyperglucosylated adhesin of non-typeable Haemophilus influenzae. Sci. Rep. 6, 39430 (2016).

50. Crivat, G., Taraska, J. W. Imaging proteins inside cells with fluorescent tags. Trends Biotechnol. 30, 8-16 (2012).

51. Lotze, J., Reinhardt, U., Seitz, O., Beck-Sickinger, A. G. Peptide-tags for site-specific protein labelling in vitro and in vivo. Mol. Biosyst. 12, 1731-1745 (2016).

52. Vandemoortele, G., Eyckerman, S., Gevaert, K. Pick a Tag and Explore the Functions of Your Pet Protein. Trends Biotechnol. 37, 1078-1090 (2019).

Table of contents graphic:

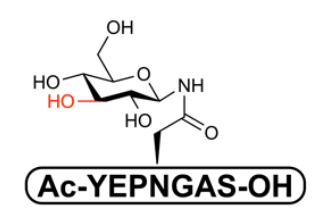

Glucopeptide

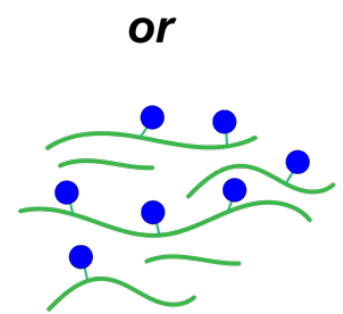

Tryptic (gluco)peptides

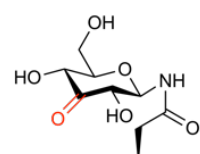

AC-YEPNGAS-OH
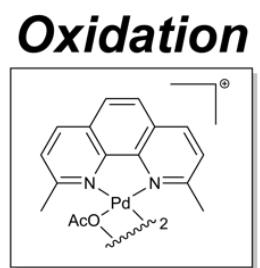

[(neocuproine)PdOAc $]_{2} \mathrm{OTf}_{2}$

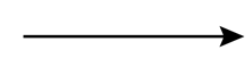

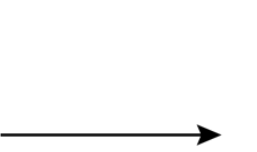

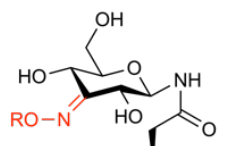

Ac-YEPNGAS-OH

\section{Oxime}

formation
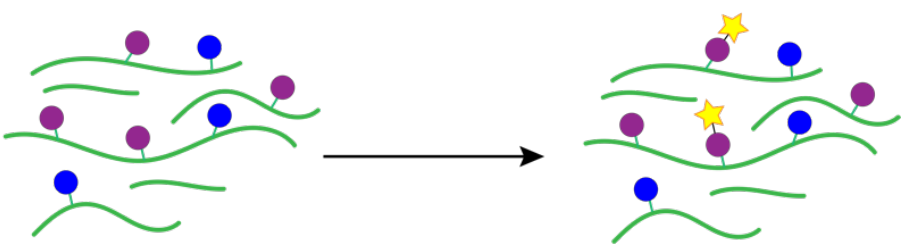